\title{
Matemáticas para todos en tiempos de la inclusión como imperativo. Un estudio sobre el programa Todos a Aprender*
}

Math for All in Times of Inclusion as an Imperative: A Study on the Program Todos a Aprender

Matemáticas para todos nos tempos da inclusão como imperativo. Um estudo sobre o programa Todos a Aprender

\section{Gloria García-Oliveros** (iD orcid.org/0000-0002-5800-7876 Julio Hernando Romero-Rey*** (iD orcid.org/0000-0002-2001-3808}

\footnotetext{
Artículo de investigación

Revista Colombiana de Educación, N.7 74. Primer semestre de 2018, Bogotá, Colombia.

Para citar este artículo: García, G. y Romero, J. (2018). Matemáticas para todos en tiempos de la inclusión como imperativo. Un estudio sobre el programa Todos a Aprender. Revista Colombiana de Educación, (74), 289-310.
}

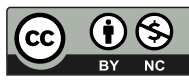

* El programa para la excelencia docente y académica Todos a Aprender es una iniciativa del Ministerio de Educación Nacional de Colombia que: "busca promover la calidad de la educación y disminuir las brechas entre lo urbano y lo rural, de modo que los estudiantes de las zonas rurales y urbanas, tengan las mismas oportunidades para el desarrollo integral de sus competencias, mediante la cualificación del ejercicio profesional de los docentes y el fortalecimiento organizacional de las instituciones educativas" (MEN, 2012)

** Magister en Filosofía. Profesora. Departamento de Matemáticas, Universidad Pedagógica Nacional. Bogotá Colombia. Correo electrónico: gloriag@pedagogica.edu.co

*** Magister en Docencia de las Matemáticas. Profesor Universidad Distrital Francisco José de Caldas, Facultad de Ciencias y Educación. Bogotá, Colombia. Correo electrónico: jhromeror@udistrital.edu.co 


\section{Resumen}

En este artículo, con referentes de Thomas Popkewitz, se analiza la problemática de la equidad, específicamente las estrategias de inclusión mediante la administración socia y normalización de los Derechos Básicos de Aprendizaje (DBA) de las matemáticas que operan el principio "Matemáticas para todos". Se realiza el análisis documental en torno a tres problemáticas ligadas a la inclusión de todos: (1) las relaciones entre administración social del Estado, educación matemática y equidad; (2) la investigación en educación matemática que se ha ocupado de la problemática de equidad e inclusión; y (3) los aparatos de administración y mecanismos de regulación de prácticas de aprendizaje de las matemáticas inclusivas. El análisis se realizó sobre dos tipos de documentos, los del campo de investigación en educación matemática y de políticas nacionales y estudios internacionales, y los de materiales curriculares y técnicas de evaluación. El análisis posibilitó concluir que la gestión de estrategias de inclusión de los DBA sigue considerando que el aprendizaje es monolítico y la gestión de homogenización del aprendizaje allí promovido vuelve a reforzar la exclusión. A partir de este análisis nos interrogamos acerca de con qué políticas del conocimiento queremos incluir a todos los estudiantes para que aprendan matemáticas.

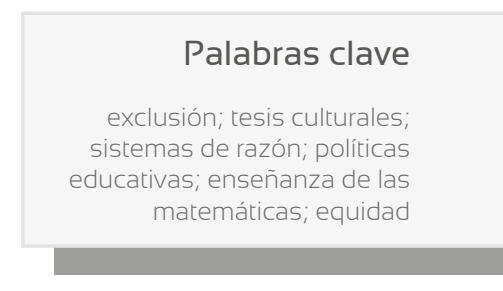

\section{Keywords}

exclusion; cultural theses; reason systems; educational policies; mathematics teaching; equity

\begin{abstract}
In this paper, we refer to Thomas Popkewitz[1] and analyze the issue of equity, more specifically the strategies of inclusion through social administration and standardization of the Basic Learning Rights (BLR) in mathematics operating under the "Math for all" principle A documentary analysis was carried out of three issues related to the inclusion of all: (1) the relationships between social administration of the State, mathematics education, and equity; (2) research in mathematics education, which has addressed the issue of equity and inclusion; and (3) management devices and mechanisms of regulation of inclusive mathematics learning practices. The [2] analysis was conducted on [3] two types of documents $\urcorner$-those from the field of research in mathematical education and national policies and international studies and those about curriculum materials and assessment techniques. [4] The analysis revealed that the management of DBA inclusion strategies still considers that learning is monolithic, and the management of homogenization of the learning promoted there reinforces the exclusion again. Based on this analysis, we raised the question [5] about which knowledge policies we wish to include when teaching
\end{abstract}

\section{Resumo}

Neste artigo, com referências de Thomas Popkewitz, analisa-se a problemática da equidade, especificamente as estratégias de inclusão através da administração social e normalização dos Direitos Básicos de Aprendizagem (DBA) das matemáticas que operam o princípio "Matemáticas para todos". Realiza-se a análise documental ao redor de três problemáticas ligadas à inclusão de todos: (1) as relações entre administração social do Estado, educação matemática e equidade; (2) a pesquisa em educação matemática que aborda a problemática da equidade e inclusão; e (3) os aparatos de administração e mecanismos de regulação de práticas de aprendizagem das matemáticas inclusivas. A análise foi aplicada sobre dois tipos de documentos: aqueles do campo de pesquisa em educação matemática e de políticas nacionais e estudos internacionais e aqueles materiais curriculares e técnicas de avaliação. A análise possibilitou concluir que a gestão de estratégias de inclusão dos DBA ainda considera que a aprendizagem é monolítica e a gestão de homogeneização da aprendizagem lá promovida reforça novamente a exclusão. A partir desta análise, nos questionamos sobre quais políticas do conhecimento queremos incluir no ensino das matemáticas.

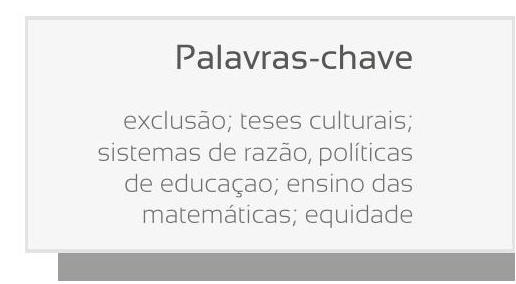




\section{Introducción}

Desde los inicios de década de los noventa, la célebre Conferencia Mundial sobre Educación para Todos (Jomtien, Tailandia, marzo de 1990) aprobó la "Declaración Mundial sobre Educación para Todos y el Marco de Acción para Satisfacer las Necesidades Básicas de Aprendizaje", y declaró como meta la universalización de la educación primaria, secundaria y superior. La universalización de la primaria debe estar dirigida a los niños que por haber nacido en una región pobre o en familias desfavorecidas se han visto privados del derecho a la educación (Organización de las Naciones Unidas para la Educación, la Ciencia y la Cultura, UNESCO, 2010). Con los auspicios de la unEsCo y el Banco Mundial se comenzó a impulsar el imperativo de la inclusión con el lema "Educación para todos" (EPT), como una de las estrategias contemporáneas más potentes para que el ideal de la universalización de los derechos individuales sea visto como una posibilidad para el funcionamiento de la gubernamentalidad neoliberal (Lopez Corsini, Henn Fabris, 2013). Una década más tarde, en el Foro de Dakar (2000), los países acordaron garantizar las condiciones y los recursos requeridos para alcanzar los objetivos de la "Educación para todos", integrando en los planes nacionales de educación el propósito de alcanzar las metas a más tardar en el año 2015.

En el 2010, la UNESCO publicó el informe "Llegar a los marginados", en el que identifica algunas de las causas profundas de la desventaja social en el ámbito de la educación, y presenta ejemplos de políticas y prácticas que apuntan a alcanzar objetivos específicos y cosechan éxitos en la lucha contra la exclusión. El propósito, como lo expresa el título del informe, es llegar a los ciudadanos marginados y preparar una respuesta eficaz que permita atender a los millones de niños de las familias de menos recursos en los países más pobres (del África subsahariana, Asia meridional y occidental, y de la región del Caribe latinoamericano) para corregir las desigualdades, la falta de equidad con programas de enseñanza primaria de buena calidad. Los programas deben poner especial atención a las competencias básicas de lectura, escritura y cálculo porque son determinantes en las posibilidades de futuro empleo. La garantía de la calidad de los programas debe verse reflejada en resultados de aprendizaje reconocidos y mensurables (p. 20).

Colombia suscribió el pacto para lograr las metas formuladas en el "Marco de acción para la satisfacción de las necesidades básicas del aprendizaje", con el Programa Todos a Aprender. Este está orientado a intervenir establecimientos educativos de primaria con bajos resultados educativos, medidos mediante las pruebas Saber y PISA, que están localizados en contextos con condiciones muy difíciles (Ministerio de Educación 
Nacional, MEN, 2012). Los establecimientos seleccionados con preferencia están localizadas geográficamente en zonas rurales caracterizadas como áreas pobres de difícil acceso, escasas o inadecuadas dotaciones referentes al proyecto pedagógico, y pésima infraestructura educativa. En el marco de la política "Cierre de brechas", el programa se propone trasformar eficazmente la calidad de los aprendizajes en lenguaje y matemáticas -en relación con logros de aprendizaje-, lo que implica mejorar las prácticas de aula que movilizan los aprendizajes de estudiantes de primaria (de transición a quinto grado). El componente de formación del profesor situado, y en paralelo, es la garantía de ingreso y permanencia en la escuela del programa. Los logros que se esperan deben reflejarse "en un futuro cercano en mejores resultados en las diferentes pruebas nacionales e internacionales [Saber y PISA] en las que participa el país" (MEN, 2012).

Cabe señalar que la modalidad de escolarización de la educación primaria en los contextos rurales colombianos es Escuela Nueva: un modelo flexible conocido como multigrado con uno o dos docentes. El modelo permite a los estudiantes la promoción entre grados, de acuerdo con su ritmo de aprendizaje, y ofrece continuidad en caso de ausencias temporales, como la producida por la integración de los niños al trabajo rural (MEN, 2012).

Inés Dusell (2000) y Thomas Popkewitz (2009) argumentan que a partir de la segunda mitad del siglo xx los principios de igualdad y libertad con los que se proclamó la expansión del sistema escolar moderno se han redefinido de tal forma que han Ilevado a la exclusión social, puesto que en una economía de mercado global y competitiva el propósito de alcanzar masivamente a la población y asegurar su ciudadanía y fuerza de trabajo productiva se ha convertido en la paradoja de la inclusión. En el caso de los países latinoamericanos, el incremento de las desigualdades sociales ha llevado a que los sistemas educativos promuevan la educación para todos mediante la individualización, maximización de oportunidades de aprendizaje y el logro académico (Dusell, 2000). La evidencia proporcionada por los sistemas internacionales comparativos de indicadores educativos (porcentaje) aporta para la formulación de medidas concretas que favorezcan acciones sobre la inclusión de grupos de personas. En este sentido, la inclusión de todos se torna un asunto de administración social calculable para identificar y ordenar la planeación de intervenciones que conduzcan a alcanzar resultados y metas estandarizadas (Popkewitz, 2009).

Para Popketwiz la equidad se entiende como el conjunto de acciones que rectifican el problema de la exclusión social (2009) con la conformación de programas que garanticen un rango de resultados de inclusión de acuerdo a estándares establecidos. Para este autor, el sistema de argumentos que relaciona la inclusión con la educación está formado por reglas, normas, 
valores que expresan lo que se clasifica como normal al mismo tiempo que lo que no lo es. Es decir que las reglas invisibilizan comparativamente lo anormal, por lo que inclusión y exclusión coexisten y no son opuestos.

Buscamos los sentidos no problemáticos dados al lema "Matemáticas para todos" y a las prácticas que constituyen el programa Todos a Aprender, en el marco de la política "Cierre de brechas". Abordar dicha problemática ha implicado preguntarnos ¿qué significa entender el lema "Matemáticas para todos" como un imperativo de inclusión? y ¿qué caracteriza las estrategias de inclusión en el programa Todos a Aprender en Colombia?

El análisis se realiza sobre dos tipos de textos. El primero, constituido por documentos del campo de investigación en educación matemática y de políticas nacionales y estudios internacionales entre el 2006 y el 2016. El segundo, compuesto por materiales curriculares y técnicas de evaluación. El análisis ha posibilitado concluir que la gestión de estrategias de inclusión de los Derechos básicos de aprendizaje con el fin de caracterizar prácticas inclusivas para todos, sigue considerando que el aprendizaje es monolítico y la gestión de homogenización del aprendizaje allí promovido vuelve a reforzar la exclusión. A partir de este análisis nos interrogamos con qué políticas del conocimiento queremos incluir a todos los estudiantes para que aprendan matemática.

En este artículo, el primer apartado describe la perspectiva de análisis, la equidad en la relación entre la administración social del Estado y el papel de la educación matemática en la producción de la inclusión. El segundo apartado analiza, en el campo de investigación en educación matemática, tres enfoques que se han ocupado del lema "Matemáticas para todos". Enseguida analizamos los movimientos de la inclusión en el contexto colombiano: estandarización y administración para aumentar el acceso y la representación de los excluidos en documentos de políticas educativas tanto nacionales como producidos por los organismos de cooperación internacional instalados en Latinoamérica. En el cuarto analizamos mecanismos de administración y normalización de la inclusión en materiales curriculares y técnicas de evaluación producidos en el programa Todos A Aprender. En la última sección reseñamos las preguntas que nos permiten formular la posibilidad de pensar de otra manera el imperativo de la inclusión en el lema "Matemáticas para todos".

\section{Sobre la perspectiva de análisis}

Comenzamos por decir que en nuestros intereses por estudiar y comprender los procesos de (in)exclusión en la escolarización de las matemáticas nos hemos movido entre los estudios empíricos en la clase de matemáticas 
para montar escenarios de aprendizaje ${ }^{1}$ inclusivos, estudios sobre el funcionamiento del currículo de matemáticas como una tecnología de gobierno, y las discusiones interdisciplinarias sobre los aportes de enfoques sociopolíticos de la educación matemática, estudios foucaultianos en educación, estudios etnomatemáticos y teorías sociales latinoamericanas.

Para comprender los modos de producción de verdad o de reconducción de la forma de accionar de las prácticas inclusivas con las matemáticas escolares, hemos realizado un estudio genealógico de las matemáticas escolares en el proyecto colonizador español en Colombia y sus mutaciones en la consolidación del Estado-nación en el siglo xx (Valero y García, 2014). Al explorar en el currículo de matemáticas el modo en que se constituye al sujeto racional cosmopolita moderno, como una forma de gobierno de la conducta de profesores y niños, encontramos que la gran narrativa de la inclusión y la promesa de un mejor futuro no solo han acompañado las prácticas educativas de las matemáticas a lo largo del siglo $\mathrm{xx}$, sino que operan clasificando y excluyendo los sujetos cuyas formas de vida no se insertan en la norma de la modernidad. La norma cultural con la que se ha formulado la tesis sobre quiénes son los sujetos deseados conjugan la matemática con el ideal del niño cognitivo universal referenciado en las teorías constructivistas del aprendizaje de las matemáticas.

En los estudios que hemos realizado en las clases de matemáticas en escuelas localizadas en contextos críticos o marginales, nos hemos encontrado a menudo con el señalamiento de malos hábitos, falta de motivación y de condiciones de aprendizaje de los estudiantes como disposiciones que impiden la inclusión. Las imágenes de gente civilizada como requisitos del progreso y los temores ante gente no reconocida como civilizada, familias en situaciones de pobreza y existencia de barras juveniles expresan los temores a los espacios culturales de pertenencia: el niño indígena en desventaja además de los niños en situación de riesgo. Estos temores transitan sutilmente en la investigación, en las políticas de escolarización para reconocer que es necesario que las poblaciones excluidas tengan oportunidades de acceso a la educación.

1 Con estudiantes de la Maestría en Docencia de la Matemática de la Universidad Pedagógica Nacional y profesores de esta universidad, la Universidad Distrital Francisco José de Caldas (Colombia) y una profesora de Aalborg University (Dinamarca) hemos realizado las siguientes investigaciones: 2007-2009. Reinventando el currículo y los escenarios de aprendizaje de las matemáticas. Un estudio desde la perspectiva de la educación matemática critica. idep, Colciencias, Universidad Pedagógica Nacional, Universidad de Aalborg (Dinamarca). Contrato 0.80 2011-013 Estudio del papel de los escenarios y ambientes de aprendizaje de las matemáticas en los procesos de inclusión en las clases. Colciencias, Universidad Pedagógica Nacional, Universidad de Aalborg, Universidad Distrital Francisco José de Caldas. Contrato 363. 
El potencial emancipador de la razón matemática ha bosquejado las condiciones para regularizar la escolarización de la matemática a través de los discursos psicológicos fundamentados en el "conocimiento de cómo se desarrolla y aprende el niño" (Walkerdine, 1995) estableciendo la regulación del desarrollo del niño normal. Parafraseando a esta autora, podemos decir que el binomio niño en desarrollo-matemática ha inscrito tesis culturales en las prácticas escolares acerca del niño que actúa y piensa desde la racionalidad de la matemática descritas por inscripciones psicológicas constructivistas que especifican las predisposiciones internas de los niños para aprender matemáticas. En esta perspectiva los procesos normalizadores ordenados según las inscripciones psicológicas definen idealmente la unidad "todo niño que aprende". Esta unidad es la que genera diferencias y temores sobre aquellos niños que invisiblemente están definidos en estos procesos como los que no aprenden (p. 12).

Como ya se mencionó, la evidencia proporcionada por los sistemas internacionales comparativos de indicadores educativos (porcentajes), en especial sobre el rendimiento en matemáticas, incluyó el aprendizaje de las matemáticas como parte sustancial de las estrategias para hacer operar el principio de la educación para todos. Las estrategias de inclusión es basan en la idea de que la educación es una necesidad básica y una aspiración social. Esta perspectiva basada en derechos se propone garantizar las condiciones mínimas asociadas al desarrollo de los sujetos en la educación con estrategias de oportunidades como el acceso y la representación de personas y grupos excluidos (Departamento de Evaluación Social, de la División Social del Ministerio de Planificación y Cooperación [Mideplan] del Gobierno de Chile, 2002) que son afectados por las desigualdades. En este contexto la equidad es el desafío para la reducción de las desigualdades.

En el contexto latinoamericano las desigualdades han dado lugar a establecer lo que el Programa de Promoción de la Reforma Educativa en América Latina y el Caribe (Preal) Ilama la perspectiva de la disminución de las brechas de equidad. Las brechas se asumen como la distancia entre la situación observada y una norma que intenta incorporar las valoraciones predominantes en América Latina, las cuales a su vez se corresponden con las aspiraciones y con las tradiciones culturales (p. 5)

Para Popkewitz (2009) la problemática de la equidad se vincula con la administración social puesto que es operacionalizada como un conjunto de acciones que rectifica el problema de la exclusión social. La estrategia es establecer cuáles actividades se pueden controlar o dirigir para lograr un aceptable rango de resultados, de acuerdo con un estándar social establecido que, en el caso del aprendizaje matemático, se puede leer con indicadores de la optimización del aprendizaje y el aumento en la 
representación de los grupos e individuos excluidos. La estrategia despliega un dispositivo que se impone a gran escala a través de prácticas, técnicas operativas que corrigen la exclusiones.

Para Popkewitz y Lindblad (2005) es necesario ampliar la noción de equidad con estudios que problematicen las distinciones y categorías de los programas inclusivos que ubican lo que está dentro de la razón (cualidades y disposiciones que el docente tiene que identificar, desarrollar y mantener en la enseñanza) y que también dicen por omisión, lo que está fuera.

Gutierrez y Dixon-Roman (2011) señalan que el discurso de cerrar las brechas en el rendimiento de las matemáticas en los Estados Unidos se ha instalado en el marco de la política económica de rendimiento de cuentas, con los aportes de la evidencia de los estudios comparativos que otorgan a las estrategias rigurosidad para continuar privilegiando la normalización de ciertos grupos. Estos investigadores coinciden con Popkewitz y Lindblad en que es necesario desestabilizar la noción de equidad al preguntarse sobre qué cuenta como matemáticas en la escolarización. Desde la perspectiva de los estudios foucaultianos explican cómo la brecha opera para sugerir que en el sistema no hay errores y oscurece las formas como se crea y sostiene la inequidad en la escolarización. El centro de la inclusión en cerrar las brechas oculta también el cuestionamiento a las normas que soportan las narrativas sobre los estudiantes marginalizados.

\section{La emergencia de las matemáticas para todos. Distinciones conceptuales y estrategias de inclusión}

En este apartado analizamos, en tres propuestas de la educación matemática, las perspectivas desde las cuales podemos construir un mapa de las distinciones conceptuales en la constitución de lo que entendemos por "todos" y de las estrategias de inclusión en el lema "Matemáticas para todos". Señalamos sus vínculos al paso de la regulación de prácticas con las matemáticas inclusivas.

Cosby (1998) describe en el grabado La templanza (1560), de Pieter Bruegel, cómo desde el siglo xvı contar y medir han estado incorporadas en las actividades desarrolladas por los oficios urbanos: cartógrafos que trazaban los mapas de los países recién descubiertos, burócratas que administraban imperios y compañías, banqueros que controlaban riquezas, artistas que desarrollaban perspectivas.

Varela (1992) reseña que a medida que se desarrollaron las relaciones de producción con el empuje de la Revolución Industrial se desencadenaron procesos de anexión y confiscación de saberes locales y artesanales para saberes más generales e industriales (p. 15). Por cuestiones de espacio en este artículo no entramos a describir el proceso de trasformación de 
los saberes en la legitimación de saberes formales, pero es claro el papel de la universidad para ejercer el monopolio de sancionar con estatuto de cientificidad solo los saberes que estas instituciones forman y aprueban.

Cline (2001) ha estudiado en la sociedad estadounidense entre 1820 y 1840, la multitud de comportamientos que ocurrían en la vida diaria y que constituyeron la mentalidad cuantitativa en la que se iniciaba la ciudadanía estadounidense en la sociedad del comercio. La alfabetización numérica apareció en el siglo xx como una actividad relacionada con la sociedad del comercio en la cual los grandes números eran portadores de valores y tenencia de capital, por ejemplo. Para Rose (1991) distintos números forman parte del gobierno democrático, puesto que es un poder calculado. Los números son intrínsecos en formas de justificación y de legitimidad de este gobierno; al mismo tiempo, hacen posible el gobierno de sí mismo cuando el ciudadano autónomo se autorregula con el poder de los números: calcula en los asuntos financieros, de salud y en la proyección de futuro.

La emergencia del lema "Matemáticas para todos" está estrechamente relacionada con los procesos de institucionalización de la educación de masas en los países occidentales: Estados Unidos y Europa o, en el decir de Keitel (1998), en países industrializados o desarrollados. Esto significa reconocer que en estos países a partir de la instalación del liberalismo, se garantizaron los principios de igualdad de derechos y de libertad de un Estado que se presenta como garantizador de los derechos de los ciudadanos.

Para Keitel, la llegada a las aulas de todos los estudiantes introdujo distintas preguntas (p. 66): ¿Las necesidades matemáticas de la mayoría determinan las posibilidades de construir propuestas que den cuenta del lema "Matemáticas para todos"? ¿Cuál es el significado de combinar unas matemáticas para los pocos y otras para la mayoría? ¿A qué tipo de necesidades sociales deben responder las matemáticas para todos, o deben responder al movimiento progresista de desarrollo psicológico del niño? ¿Las matemáticas para la mayoría deben estar relacionadas con las finalidades dadas por el tipo de educación generalista que planteaba la masificación de la escuela: formación para la vida laboral?

Las respuestas giran en torno a la preservación de la matemática orientada a la teoría para estudiantes que proseguían a sus estudios en la universidad. Keitel relata que los interrogantes y las decisiones de la selección de las matemáticas para todos visibilizaron el carácter elitista de la perspectiva de la matemática como teoría. Por su parte, las peguntas sobre a qué se refería el "todos" ocuparon una posición secundaria y marginal en la medida en que los presupuestos de las teorías del constructivismo y de las teorías de aprendizaje de las matemáticas garantizaban la visión del estudiante como sujeto universal, caracterizado por la "actividad cognitiva". De cierta forma estos argumentos funcionan como metáfora del modo como Descartes y Leibniz consideraban que se daban en la mente los "principios internos" que 
necesitamos para entender los objetos o para percibir las propiedades de la matemática, si bien para alcanzar las verdades matemáticas no necesitamos el concurso de los sentidos ni de la experiencia.

Si las matemáticas para todos debían responder al tipo de formación, la selección obligaba a incluir las matemáticas aplicadas que se encontraban en el mundo. Para la comunidad inglesa todo lo que era preciso hacer era investigar y definir las habilidades necesarias para las diversas ocupaciones laborales. El célebre Informe Cockcroft (1982) publica los estudios desarroIlados en la Gran Bretaña y Gales sobre habilidades numéricas (numeracy en inglés) y las necesidades futuras que en cultura matemática se requería introducir a la escuela inglesa. El concepto numeracy tiene como referente la necesidad de pensar de manera cuantitativa en el mundo moderno. El informe amplía el uso de las capacidades numéricas en las nuevas tecnologías.

La orientación dada en los estudios y discusiones a la democratización de la escolarización de las matemáticas constituyó una de las verdades dominantes en lo que restaba del siglo xx: el cuestionamiento a la matemática académica como fuente exclusiva de la enseñanza de esta disciplina creó el movimiento por las relaciones con los espacios de los saberes matemáticos en las prácticas sociales.

La orientación dada por Hans Freudental (matemático y educador, miembro del grupo holandés ow\&oc, Vakgroep Onderzoek Wiskunde Onderwijs, 1970) al lema "Matemáticas para todos y para cada uno" -conocido como la propuesta de ıwo- se estableció en términos relacionales con la exigencia democrática de la educación dentro del sistema político holandés. El punto de partida de la propuesta, que llamó desarrollo educativo, son experiencias de enseñanza de las matemáticas. Su construcción parte de un material que se entrega a los profesores, quienes lo reelaboran hasta adaptarlos de la mejor manera posible a una actividad de aprendizaje (Keitel, 1998). Vincula tanto a los profesores como los textos escolares, que en su gran mayoría han sido adoptados en Holanda como posibilidades reales para realizar cambios en la escuela para lograr una matemática para todos.

Las experiencias para incluir a todos y cada uno están orientadas por las siguientes preguntas: ¿Cómo aprende el niño las matemáticas? ¿Por qué las aprende? y ¿Qué es lo que aprende? Como matemático, subraya la importancia no solo de la actividad de hacer matemáticas para llegar a los productos, sino de enseñarla como eje fundamental en los currículos de matemáticas. Supone que la matemática como actividad humana es una tarea para resolver problemas de la realidad. Con esta visión propone que las matemáticas escolares se puedan pensar para ser útiles, pues la realidad del mundo puede ser organizada de acuerdo con las matemáticas, por lo que la organización de las actividades de aprendizaje implica trabajar temas de la realidad y temas matemáticos (p. 3). El sentido de organización, de hacer matemática, lo denomina "matematizar". 
Las formas de matematización caracterizan la comprensión de los estudiantes y tipifican el proceso de aprendizaje concebido en niveles de desarrollo. De esta manera la norma que regula la actividad de hacer matemática en la comunidad de matemáticos se establece como norma cultural para la enseñanza. La matematización resulta el modelo ideal, fundamentado en la estructura invariante de niño cognitivo universal, en la producción de comportamientos en el aprendizaje de las matemáticas. La matematización se torna en la base de la hipótesis que sostiene el marco teórico de las competencias matemáticas definidas en PISA (Rico, 2007) pues se toma como una herramienta que al organizar realidades matemáticamente relevantes para el bienestar económico y social del mundo, es la que caracteriza a los ciudadanos del mundo (Rico, 1997). De esta manera la matematización pasa a regular la población escolar.

Entre los autores que contribuyeron a desarrollar una teorización y una propuesta desde una perspectiva cultural, se destaca Alan Bishop (2000), quien introdujo preocupaciones sobre quiénes son todos los estudiantes. Su trabajo se desarrolló un poco más tarde que el de Freudenthal, y cuando ya estaba en circulación la propuesta de etnomatemática, Bishop elaboró una crítica al colonialismo cultural de las matemáticas occidentales que se presupone en las perspectivas tradicionales del currículo de matemáticas. Destacó a su vez el poder de las matemáticas occidentales para enseñarse sobre la hipótesis de neutralidad y universalidad, y resalta la diversidad de formas culturales de la matemática aportada por los estudios del programa de etnomatemáticas. Desde esta perspectiva, reconoció la necesidad de reconceptualizar el currículo con las prácticas matemáticas que se desarrollan en todas las culturas: contar, localizar, medir diseñar, explicar y jugar para satisfacer las necesidades de todos los alumnos menores de 16 años. Esta reconceptualización puede dar respuesta a los Ilamados alumnos de riesgo, que las investigaciones sugieren como portadores de dificultades, entre quienes se incluyen estudiantes pertenecientes a familias desfavorecidas, así como aquellos que experimentan conflictos culturales y lingüísticos (p. 37).

La llegada de estos alumnos a las aulas de matemáticas comenzó a ser estudiada desde una perspectiva sociológica e intercultural, pues la mayoría portan una entidad colectiva: ser emigrantes (Planas, 2003, p. 62). Los estudios de Candice Morgan, Nuria Planas, Guide de Abrue y Tony Cline comienzan a mostrar que el aula de matemáticas no es solo un escenario de prácticas y actividades matemáticas científicas; es un terreno normativo de relaciones sociales donde valores y valoraciones sociales entretejen complicadas relaciones de poder (p. 58) entre prácticas matemáticas y participantes involucrados. Los estudios en la clase de matemáticas, con estudiantes plurales culturalmente revelan que la comprensión de las normas de actuación matemática causan diferencias en las estructuras de

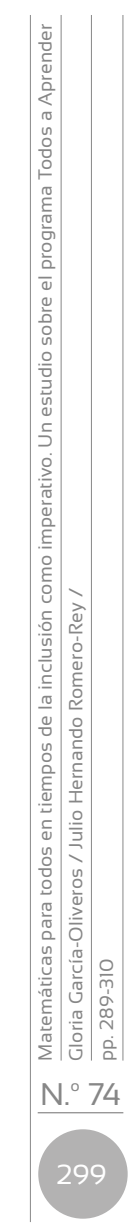


participación que los estudiantes interiorizan como algo normal. Planas (2003) pone de relieve el cuestionamiento a la relación entre calidad y equidad en aulas plurales. La calidad supeditada a las matemática para pocos genera que las oportunidades de comunicación en la clase dependan de la posiciones de los participantes relacionadas con la valoración de prácticas y de personas, y no solo de los contenidos matemáticos.

Planas reseña cómo los recientes movimientos de reforma por la igualdad de oportunidades en educación matemática han generado detractores que critican los peligros de una enseñanza de las matemáticas que incluya a todos. Al igual que en el movimiento de las matemáticas para todos de mediados del siglo xx, como hemos reseñado, los temores a rebajar la calidad de las matemáticas para unos pocos persisten y plantean la dicotomía entre dos principios fundamentales: el de la calidad y el de equidad (Planas, 2003, p. 73). Por otra parte, los valores de equidad relacionados con la igualdad de oportunidades que tienen que ver con valorar a todos los alumnos por igual implica considerar el aula de matemáticas como espacio social donde sea posible la negociación del conocimiento.

Con referentes de la etnomatemática, Knijnik (1996) ha cuestionado la política del conocimiento matemático desde la que se han invisibilizado manifestaciones culturales distintas a los grupos socialmente legitimados para producir el conocimiento matemático científico. Con el estudio de los saberes regionales útiles en la toma de decisiones en las prácticas del cultivo que desarrollan los campesinos del Movimiento Sin Tierra, propone referirse a las matemáticas como artefactos culturales conectados con formas de vivir y de producción de significados en grupos sociales. Señala esta autora que en la contemporaneidad la particular exclusión por el conocimiento matemático, que no es nueva, al producir la exclusión social desconoce, por ejemplo, que en la lucha por la tierra en Latinoamérica la matemática, no solo referida a cuentas, es necesaria en la organización, producción y comercialización de la agricultura.

Lubienski y Gutiérrez (2011) sitúan el discurso de la equidad en los Estados Unidos desde la estrategia conocida como "cerrar brechas" (en inglés, game gazing) con la cual se busca alcanzar la educación para todos. La estrategia es deudora del discurso de los logros educativos y en la actualidad ha tomado fuerza con los estudios comparativos internacionales provenientes de pruebas internacionales como PISA. Este enfoque tiene como referente la necesidad de controlar los niveles cada vez mayores de población como resultado de la extensión de la educación escolarizada en Norteamérica. Con referentes que proceden de la concepción del funcionamiento de la escuela como empresa comercial o industrial, Tyler formula el currículo como estrategia de control de la población escolar. El punto central es 
establecer con precisión la formulación de los objetivos de aprendizaje en términos de comportamientos explícitos y claramente definidos para saber con precisión si los resultados esperados se alcanzan (Da Silva, 2001).

Es interesante observar cómo el currículo como modelo de control tuvo una gran acogida en las políticas educativas para gestionar la extensión y universalización de la educación primaria y básica en las reformas que se adelantaron en las décadas de los sesenta y setenta del siglo xx en América Latina. Con esta estrategia la evaluación de los rendimientos de los estudiantes, Ilamados desempeños en el lenguaje de las competencias, se constituye en la herramienta ideal para lograr la educación de todos. Pero, en la contemporaneidad la evaluación de los desempeños trasciende el control de la clase para regular la población escolar a partir de los llamados sistemas de evaluación nacional e internacional con los estudios comparativos (Klein, 2013). Con estos sistemas es posible mostrar el desplazamiento de la evaluación como tecnología disciplinar, que controla espacios y tiempos, y constituir la subjetividad hacia una tecnología política de vigilancia y normalización constante sobre la población para garantizar la inclusión de todos en la escuela.

El movimiento hacia la población exige la estrategia de estandarización que al mismo tiempo que visibiliza el compromiso social y político para cumplir que todos los niños aprendan matemáticas, establece el centro como métrica para comparar la evaluación del rendimiento de los estudiantes en pruebas.

\section{Todos a Aprender (PTA): ¿una inclusión paradójica?}

De manera general podemos decir que los asuntos sobre la equidad comenzaron a ser incorporados en las políticas colombianas mediatizadas por las demandas formuladas por organismos internacionales como la unESCO, el Banco Mundial, la oea y la Cepal (Martínez, 2004) entre los años sesenta y setenta con las políticas de expansión. En palabras de Martínez, la expansión se inició con el proyecto de universalización que buscaba homogeneizar, bajo ciertos criterios mininos, la mayor cantidad de población. Para este autor, con esta reforma se inició el proyecto incluyente al buscar involucrar a la mayoría de la población en la escolarización.

Desde la década de los noventa Colombia acogió los principios del neoliberalismo en educación, lo que ha significado adoptar la agenda global universal para llevar a cabo procesos educativos con calidad y equidad. También ha significado acoger las recomendaciones y demandas formuladas por los organismos internacionales instalados en la región latinoamericana después de la Segunda Guerra Mundial, más conocidos como organismos de cooperación técnica (Orealc/Unesco, Organización 
de Estados Americanos [oet], Programa de Promoción de la Reforma Educativa en América Latina y el Caribe (Preal], Banco Mundial [вм], Banсо Interamericano de Desarrollo [BID]).

En lo que va corrido del siglo xxı estos organismos ${ }^{2}$ han generado un gran volumen de investigaciones para identificar el conocimiento útil y necesario para comprender los factores que aumentan o disminuyen la representación y el acceso de los grupos sociales y los individuos a programas de rectificación de la exclusión. Los estudios sobre los factores del aprendizaje, junto con la medición de las desigualdades de oportunidades hasta la relación entre profesores excelentes y mejores aprendizajes, se constituyen en el conocimiento útil y garantizado para diseñar programas más eficientes en la generación de la equidad en la región. En palabras de Popketwiz (2005), una característica de estas investigaciones es la producción de un conocimiento comparativo basado en indicadores entre categorías individuales y entre poblaciones o países, y precisar las diferencias Ilamadas brechas en la esquematización de la noción de equidad. La reducción de la brecha requiere de fijar un centro para producir los resultados esperados desde el cual normalizar los rendimientos.

Colombia inscribe los compromisos de la declaración sobre EPT en lograr que el sistema educativo forme el capital humano para aumentar la productividad en el discurso del Plan Nacional de Educación 20062016. En este se establece la inclusión como uno de los ejes que define el horizonte por seguir durante la década:

Construir una sociedad más equitativa, humana y justa, supone garantizar la total inclusión de mujeres y hombres en la dinámica social y desarrollar estrategias específicas para que las personas o los grupos de la población que se encuentran en condiciones precarias o en situación de desventaja, puedan mejorar su situación actual. Todos los esfuerzos que el país realice, orientados a mejorar la situación de la población colombiana en general, deben afectar positivamente tanto a hombres como a mujeres; en esta medida, se irán cerrando las brechas de género, las brechas sociales y las brechas regionales. (Departamento Nacional de Planeación, tomo 2, 2007, p. 34).

La idea central de la política es cerrar brechas para garantizar la inclusión social de todos a través de estrategias específicas dirigidas a personas en riesgo: caracterizadas por condiciones precarias. Estas estrategias deben mostrar su efectividad positiva. Una de las prioridades es cerrar las brechas en la educación, concebida como la herramienta que potencia el desarrollo del recurso humano necesario para el desarrollo económico sostenible al mismo tiempo que reduce la pobreza (Martínez, 2004).

2 El Banco Mundial, por ejemplo, ha generado desde 2003 la serie "Foro sobre desarrollo de América Latina", que cuenta en la actualidad con más de 30 investigaciones. 
La reducción de las brechas en educación se identifica en el Plan de Acción del documento de Jomtien con el reconocimiento de los Derechos Básicos del Aprendizaje. Este reconocimiento en gran medida tiene como referente los resultados de la investigación

... sobre la comprensión de qué aprenden los estudiantes y cómo lo hacen, puesto que es un tema de gran interés para las políticas interesadas en asegurar los derechos humanos para reducir desigualdades a fin de mejorar los resultados de la vida individual, la competitividad, el crecimiento económico y los resultados del desarrollo. (Vegas, Petrow, 2008, p. xxIII).

Para estos investigadores, el desafío de la región es adoptar una visión institucional para lograr una buena coordinación de las funciones y responsabilidades de todos los participantes y asegurar cerrar las brecha con la calidad del sistema educativo. Merchán (2015) señala que la política "Cerrar brechas" endosa la responsabilidad a la escuela y los profesores, al mismo tiempo que configura la evaluación como parte de un dispositivo que posee una doble cualidad: mejorar la calidad de la educación y asegurar el control sobre lo que aprenden los estudiantes. De esta manera se agregan indicadores institucionales en la esquematización de la equidad; estadísticas relacionadas con los rendimientos de eficacia de los centros escolares.

El Programa Todos a Aprender se inscribe como una necesidad para alcanzar las metas de los países en desarrollo, como en el caso de Colombia, para insertarse con éxito en la sociedad del conocimiento del siglo XXI (Ministerio de Educación Nacional, MEN, 2012). La presentación del programa reseña el esfuerzo realizado en pro de lograr la calidad modificando los esquemas de evaluación para conocer con precisión el nivel logrado por los estudiantes en los diferentes establecimientos educativos. A este esquema se han sumado los aportes de los resultados dados por la participación en las pruebas internacionales que sirven como referentes para comprender lo que se debe "transformar en un sistema educativo que no garantiza los aprendizajes que requieren los ciudadanos para participar exitosamente en la sociedad del siglo xxı" (p. 4).

Junto al diseño e implementación del programa Todos a Aprender, el MEN (2012) fija el índice sintético de calidad (ISCE) COmo herramienta que permite comprender cómo está la calidad educativa en los colegios en relación con otros establecimientos educativos de su región. Este índice se determina entre los distintos resultados por los desempeños de los estudiantes de una institución en las pruebas externas, comparados con los resultados en las pruebas del año anterior, en relación con el ambiente escolar y con las tasas de aprobación del año escolar. El reporte de los 
indicadores de cada uno de estos elementos se entrega a los colegios para formular planes de mejoramiento; uno de los retos es reducir el porcentaje de estudiantes en el nivel deficiente.

La meta de alcanzar la equidad se diseña con la lógica de la estandarización de los Derechos Básicos de Aprendizaje para generar prácticas desde las que se promuevan la inclusión. Estas prácticas dan por supuesto que producen el acceso y la distribución en la igualdad de oportunidades para aprender. El programa introduce un modo de vigilancia directa de las prácticas de innovación inclusiva a través de una serie de prácticas denominadas de acompañamiento que involucran el "equipo de formadores" conformado por tutores y docentes del establecimiento educativo focalizado por el programa. Define como uno de los componentes del programa la formación situada para "formar a los docentes en los aspectos disciplinares y didácticos a través del acompañamiento en el aula por parte de los docentes tutores" (MEN, 2012). El programa se concentra en cerca de tres mil establecimientos ubicados en las condiciones más difíciles del país. Los objetivos para el programa se fijan como lograr que "más del $25 \%$ de los estudiantes de estos establecimientos educativos asciendan de nivel al menos en las áreas de Leguaje y Matemáticas en las pruebas Saber de $3^{\circ}$ a $5^{\circ}$ aplicación año 2014" (p. 6).

Para lograrlo, señala que es indispensable mejorar las prácticas en el aula. Para alcanzar los resultados esperados se anota la necesidad de contar con secuencias didácticas que contengan una descripción clara de los objetivos para cada sesión de trabajo y con materiales precisos para los docentes desde los cuales se presenten los problemas que típicamente tienen los estudiantes y pistas para resolverlas. El problema de este programa es que al ofrecer las prácticas inclusivas como un modo de salvamiento de los niños en situaciones de riesgo, evitando que contribuyan a la exclusión, responde a las posibilidades de inclusión con el esfuerzo personal y voluntario de los niños. Y como hemos reseñado, este esfuerzo se traduce en las posibilidades de participación en la clase de matemáticas. El fruto de los esfuerzos de cada niño para asentar la lógica de inclusión en un programa basado en la meritocracia y en la igualdad de lo que se aprende hace que emerjan, de nuevo, las viejas desigualdades de logros.

Aunque el programa ha buscado integrar diversos principios de igualdad educativa (igualdad de enseñanza, igualdad de contenidos, centrados en las capacidades de todos para aprender matemáticas), debemos cuestionar tal lógica pues hemos ilustrado las sombras que han acompañado estos principios en los estudios y esfuerzos para lograr una educación matemática para todos. En el 2015 la eficiencia del PTA logra la reducción de la brecha entre Colombia y Chile (MEN, 2015). Esto permite predecir que, si el programa sigue manteniendo constante su efectividad, al 2024 
la brecha será de 86 puntos frente a Chile. Igualmente se analizan las predicciones de las mejoras de resultados de desempeños de Lenguaje, Matemática y Ciencias en las pruebas Saber y PISA.

Para Grek (2009) esta estrategia de equidad se basa en un gobierno por comparación, puesto que el discurso construye su propuesta a través de estrategias comparativas de indicadores que tienden a imponer naturalmente respuestas similares en contextos nacionales o internacionales diferentes. Parafraseando a este autor podemos decir que los efectos de este discurso comparativo consisten en construir un espacio de competitividad y cohesión, caracterizado por la comparación validada por indicadores que hacen posible la conmensurabilidad entre los espacios educativos. La historia de estos espacios es tejida por la estadística, desde la cual se establecen equivalencias entre la estructura administrativa para la equidad y los procesos de estandarización y comparación.

\section{Los aparatos de clasificación para eliminar la exclusión y las inequidades}

Para Walkerdine (1995) los aparatos de clasificación son básicos para la posibilidad y efectividad en las formas de aprendizaje de un contenido concreto puesto que especifican la producción de los procesos de adquisición del contenido, precisando lo que está y lo que no está permitido, lo que se considera válido y al mismo tiempo lo que no. Las teorías de aprendizaje de las matemáticas han estado comprometidas en fijar normas para hacer objetivo el conocimiento y en la construcción de las nociones que formalizan el sentido de lo objetivo del conocimiento (Barragán, 2011) en el lenguaje de las competencias y los estándares. El uso normativo de modelos de aprendizaje para secuenciar las actividades da lugar a constituir lo que Walkerdine (1995) Ilama aparatos que producen la verdad sobre la posibilidad y la eficacia del aprendizaje. Se ofrecen en términos de aparatos administrativos para la provisión de técnicas de regulación social encaminadas a generar la esperanza de ningún niño excluido. El ensamblaje de los aparatos de control y clasificación del aprendizaje incluye desde la ubicación en el espacio del aula, los tiempos para el aprendizaje, especificaciones sobre los exámenes y la evaluación, registros de clase, fichas de trabajo y diseños de clase, puesto que son lugares de producción del sujeto.

En el TPA, la producción de estos aparatos ocupa un lugar esencial en la administración de la producción de la inclusión de todos, puesto que son orientaciones para el profesor como gestor del aprendizaje. El programa en once documentos Ilamados Derechos básicos de aprendizaje, para los respectivos once grados de la educación básica y media, establece el 
contenido matemático; estos se postulan como portadores casi que de una nueva visión sobre el aprendizaje de las matemáticas, lo que supondría una nueva organización de los contenidos matemáticos asociada a nuevas experiencias de aprendizaje para que los profesores se vean obligados a enfrentar innovaciones. En lo que sigue, por cuestiones de espacio, analizamos en el texto para grado primero la propuesta de los Derechos básicos relacionada con el aprendizaje del sistema de numeración decimal. Cada texto es acompañado con la serie titulada Área de Matemáticas. Mallas de aprendizaje (MEN, 2015).

El texto Derechos Básicos de Aprendizaje matemáticas grado $1^{\circ}$ establece como los dos objetivos:

1. Sabe contar de 0 a 99 [...]También contar de dos en dos o de diez en diez [...] Si ve un número puede decir su nombre y si escucha el nombre del número lo puede escribir [...].

2. Puede determinar cuántos elementos hay en una colección de menos de 100 elementos. (MEN, 2010, p. 1).

La presentación del sistema siguiendo el orden de la serie numérica preserva la forma tradicional del conteo escolar, de uno en uno, como estrategia privilegiada para avanzar por partes en el conocimiento del sistema. En esta perspectiva no se reconocen los conocimientos numéricos acerca del conteo de los niños en sus participaciones en prácticas sociales con las matemáticas. El uso oral del conteo en las prácticas de repartir, de comparar y de igualar han aproximado a los niños al conteo como una herramienta que les permite tomar decisiones en su vida; mientras en la escuela debe esperar el orden establecido para contar, por ejemplo, de dos en dos.

Por su parte el texto Área de matemáticas. Mallas de aprendizaje, organizado en tres apartados (Red de conceptos, Progresión del aprendizaje y Consideraciones didácticas) para cada grado, establece las actividades y las normas para el aprendizaje. Afirmaciones como "... a partir del juego como estrategia pedagógica [...]" fijan las características con las que se espera despertar el interés, la curiosidad y la resolución de problemas en los niños de grado primero. Al mismo tiempo formula que se espera que los niños "usen con sentido las matemáticas de la numeración [...]". La construcción del camino del conocimiento se conecta de nuevo con la selección de nociones matemáticas convencionales que forman el centro del aprendizaje en matemáticas, lo que ha hecho posible centrarse en los principios psicológicos, capacidades o disposiciones atribuidas al desarrollo del niño universal: en este caso la disposición para el juego se torna en una tesis cultural sobre el modo de vida de los niños en el aula de matemáticas. La propuesta deja sin analizar las tesis culturales tradicionales de la escolarización; por lo tanto, no cuestiona las distinciones y las categorías que enuncian la unidad del todo. 
En el apartado "Progresión de aprendizaje" la sigla DBA se traduce como "Objetivo de aprendizaje". La consecución de este objetivo se plantea en sub-objetivos que trazan el camino de lo sencillo a lo complejo: reconoce, explica, construye. Esta forma de plantear la organización en la consecución del objetivo del aprendizaje se asemeja a la teoría del aprendizaje acumulativo de Robert Gagné, que analiza las habilidades disgregándolas en sub-habilidades ordenadas Ilamadas jerarquías de aprendizaje. En el caso del objetivo "Reconoce en sus actuaciones cotidianas posibilidades del uso de los números y las operaciones", la jerarquía implicada en la solución exige por parte del maestro caracterizar las habilidades y sub-habilidades que identifican las capacidades para usar que se supone el niño posee.

La teoría de la jerarquía de aprendizaje fue validada en experimentos con los niños para establecer la rapidez de ejecución de algoritmos enseñados (Resnick y Ford, 1990). Los aparatos que reproducen las verdades sobre el desarrollo del aprendizaje reducen al niño al sujeto unitario poseedor de capacidades con las que adquiere conocimiento. La propuesta sigue siendo una práctica cuyo objeto es el niño universal individualizado en desarrollo. En el decir de Walkerdine, el papel de la práctica educativa con presupuestos transformativos sigue siendo una cuestión sin resolver, pues hemos visto que la exclusión está encubierta en la producción de la inclusión. Por lo tanto las prácticas efectivas para la inclusión son más contradictorias de lo que enuncian y sugieren las investigaciones y la esquematización de la equidad.

\section{A modo de cierre}

Podemos decir que la problematización de la equidad es una construcción inacabada que nos ha permitido penetrar en esas zonas de sombras de la equidad en el enfoque para cerrar la brecha, y cuestionar la suposición de que nada está errado en la escolarización de las matemáticas. En los estudios que hemos realizado ${ }^{3}$ empezamos por cuestionar si es en la misma escolarización que ha producido injusticias y exclusiones en la que queremos incluir a los excluidos. Analizar las condiciones por las cuales el conocimiento matemático se torna normativo nos ha mostrado las razones por las cuales cualquier cambio tiene que pasar por cuestionar la producción de la normatividad que lo deviene como saber escolarizado, pues en la actualidad parece difícil seguir sosteniendo que las voces que caracterizan los diversos grupos de alumnos no forman una sola unidad

3 Los estudios, junto con las investigaciones realizadas, se han concretado en la producción de ocho tesis de la Maestría en Docencia de la Matemática de la Universidad Pedagógica Nacional, Colombia. 
reducida a las categorías de clase, raza o género se constituyen en formaciones culturales que crean posiciones históricamente constituidas que los ubican como sujetos (García, 2016).

\section{Referencias}

Barragán, B. (2011). Experiencia y narración: ensayo sobre el conocimiento escolar como campo de subjetivación. Revista Virtual Universidad Católica del Norte, 32, 1-18. Recuperado de http://revistavirtual.ucn. edu.co/.

Bishop, A. (2000). Enseñanza de las matemáticas: ¿cómo beneficiar a todos los alumnos? En N. Gorgorió, J. Deulofeu y A. Bishop (eds.). Matemáticas y educación. Retos y cambios desde una perspectiva internacional. Barcelona: Grao.

Cline, P. (2001). The emergence of numeracy. En L. A. Steem (ed.). Mathematics and democracy. The case for quantitative literacy. United States of America: National Council on Education and the Disciplines. Washington, Estados Unidos: Library of Congress.

Colombia, Ministerio de Educación Nacional [MeN]. (2012). Programa Todos a Aprender: para la transformación de la calidad de la educación. Bogotá, Colombia.

Colombia, Ministerio de Educación Nacional [MEN]. (2015). ¿Qué quiere decir Colombia la más educada de Latinoamérica? Bogotá, Colombia. Recuperado de http://mineducación.gov.co/.../articles -.349925.

Colombia, Ministerio de Educación Nacional [MEN]. (2010). Derechos básicos de aprendizaje. Grado $1^{\circ}$. Recuperado de http://www.colombiaaprende.edu.co/html/micrositios/1752/articles-349446_genera_dba.pdf.

Colombia, Ministerio de Educación Nacional [MEN]. (2015) Malla de aprendizaje. Grado $1^{\circ}$. Recuperado de http://aprende.colombiaaprende. edu.co/es/node/89839.

Chile, Ministerio de Planificación y Cooperación de Chile [Mideplan]. (2002). Seminario desigualdad y reducción de brechas de equidad sistematización de tópicos principales. Recuperado de http://www. cepal.org/noticias/noticias/5/11215/SistSemMayo2002.pdf.

Crosby, A. W. (1998). La medida de la realidad: la cuantificación y la sociedad occidental. Barcelona: Crítica.

Da Silva, T. T. (2001). Espacios de identidad. Nuevas visiones sobre el currículum. Barcelona: Octaedro.

Departamento Nacional de Planeación [DNP]. (2007). Plan Nacional de Desarrollo 2006-2010. Estado Comunitario: desarrollo para todos (tomo 2). Recuperado de https://www.dnp.gov.co/Plan-Nacional-de-Desarrollo/ PND\%202006-2010/Paginas/PND-2006-2010.aspx. 
Dussel, L. (2000). La producción de la exclusión en el aula. Una revisión de la escuela moderna en América Latina. En X Jornadas LOGSE, La escuela y sus agentes ante la exclusión social. Granada, España.

España, Ministerio de Educación y Ciencia. (1982). Informe Cockcroft. Madrid, España.

Gagné, R. (1998). Learning hierarchies. Educational Psychologist.

García, G. O. (2016). Prácticas culturales de consumo: limitaciones y posibilidades en la movilización de la relación diversidad, educación matemática y subjetividades juveniles. Revista Educação Unisinos, 20(3), 339-347. Recuperado de http://revistas.unisinos.br/ index.php/educacao/issue/view/566.

Grek, S. (2009). Governing for number: The PISA effects in Europe. Journal of Education Policy, 4(1), 23-37.

Gutiérrez, R. y Dixon-Roman, E. (2011). Beyond gap gazing: How can thinking about education comprehensively help us (re) envision mathematics education? En B. Atweth, M. Graven, P. Valero y W. Secada (eds.). Mapping equity and quality in mathematics education. Nueva York: Springer Dordrecht Heidelberg.

Keitel, C. (1998). ¿Cuáles son los objetivos de las matemáticas para todos? Revista de Estudios del Curriculum, 1(4), 59-81.

Klein, R. (2013). A avalicao da aprendizagem como estrategia de Inclusão escolar. En Eli T. Heenn Fabris, Rejano Ramos Klein (eds.) Inclusão e Bipolitica. Estudios foucaultianos. Belo Horizonte: Autentica.

Knijnik (1996). As novas modalidades de excluso social. Trabaljo, conocimiento e Educação. En Reuniao Anual de Anepd, Caxambu. Brasil.

López Corcini, M. y Fabris, H. E. (2013). Inclusão \& Educação. Colecão temas \& educação. Belo Horizonte: Autentica.

Lubiensky S. T. y Gutiérrez, R. (2008). Bridging the Gaps in Perspectives on Equity in Mathematics Education. Journal for Research in Mathematics, 39(4), 365-371.

Martínez, A. (2004). De la escuela expansiva a la escuela competitiva. Dos modos de modernización en América Latina. España: Anthropos.

Merchán, J. F. (2015). ¿Evaluación o inculpación? Revista de la Asociación de Sociología de la Educación, 8(2), 223-236.

Organización de las Naciones Unidas para la Educación, la Ciencia y la Cultura [Unesco] (1990). Declaración Mundial sobre Educación para Todos y Marco de Acción para Satisfacer las Necesidades Básicas de Aprendizaje. Tailandia: Jomtien.

Organización de las Naciones Unidas para la Educación, la Ciencia y la Cultura [Unesco] (2010). Llegar a los marginados. Informe de seguimiento de la EPT en el mundo. Educación para Todos. París, Francia: UNESCO. 
Planas, N. (2003). El contrato social en el aula de matemáticas: episodios en torno a la noción de estatus. Boletim 41. Publicacao Do grupo de Estudos e Pesquisas em Educacao Matematica, GEPEM.

Popkewitz, Th. y Lindblad, S. (2005). Gobernación educativa e inclusión y exclusión social: dificultades conceptuales y problemáticas en la política y en la investigación. En L. Julián (ed.). Paradigmas de gobernación y de exclusión social en la educación. Barcelona, España.

Popkewitz, Th. (2006), La escolaridad y la exclusión social. Anales de la Educación Común, 2(4). Recuperado de http:// www.ugr.es/ force/ congreso/materiales/popkewitz.pdf

Popkewitz, T. (2009). El cosmopolitismo y la era de la reforma escolar. Madrid, España: Morata.

Programa de Promoción de la Reforma Educativa de América Latina y el Caribe [Preal] (2006). Educación y brechas de equidad en América Latina. Recuperado de http://www.mineducacion.gov.co/cvn/1665/ articles-127007_archivo_pdf.pdf.

Resnick, L. y Ford, W. (1990). La enseñanza de la matemática y sus fundamentos psicológicos. Madrid, España: Paidós Ibérica.

Rico, L. (1997). Los organizadores del currículo de matemáticas. En L. Rico, E. Castro, M. Coriat, A. Marín, L. Puig, M. Sierra y M. Socas (eds.). La educación matemática en la enseñanza secundaria. Madrid: Ice-Horsori.

Rico, L. (2007). La competencia matemática en PISA. PNA, 1(2), 47-66.

Rose, N. (1991). Governing by numbers: Figuring out democracy. Accounting Organizations and Society, 16, 673-692.

Valero, P. y García, G. (2014). El currículo de las matemáticas escolares y el gobierno del sujeto moderno. Bolema, 28(49), 491-515.

Varela, J. (1992). Tiempo y espacio. Revista de Educación, 298, 7-29.

Vegas, E. y Petrow, J. (2008). Incrementar el aprendizaje estudiantil en América Latina. El desafío para el siglo xxı. Serie Banco Mundial. Washington: Banco Mundial, Ediciones Mayol.

Walkerdine, V. (1995). Psicología del desarrollo y pedagogía centrada en el niño: la inserción de Piaget en la educación temprana. En J. Larrosa (ed.). Escuela, poder y subjetividad. Madrid, España: Ediciones de la Piqueta. Cruz Verde. 